\title{
Adaptive Segmentation-Based Symbolic Representations of Time Series for Better Modeling and Lower Bounding Distance Measures
}

\author{
Bernard Hugueney ${ }^{\star}$ \\ Universitè PARIS-DAUPHINE \\ LAMSADE Place du Maréchal de Lattre de Tassigny \\ 75775 PARIS CEDEX 16 \\ bernard.hugueney@lamsade.dauphine.fr
}

\begin{abstract}
Time series data-mining algorithms usually scale poorly with regard to dimensionality. Symbolic representations have proven to be a very effective way to reduce the dimensionality of time series even using simple aggregations over episodes of the same length and a fixed set of symbols. However, computing adaptive symbolic representations would enable more accurate representations of the dataset without compromising the dimensionality reduction. Therefore we propose a new generic framework to compute adaptive Segmentation Based Symbolic Representations (SBSR) of time series. SBSR can be applied to any model but we focus on piecewise constant models (SBSRL0) which are the most commonly used. SBSR are built by computing both the episode boundaries and the symbolic alphabet in order to minimize information loss of the resulting symbolic representation. We also propose a new distance measure for SBSRL0 tightly lower bounding the euclidean distance measure.
\end{abstract}

\section{Introduction}

Time series are easily collected in huge amounts only limited by the sampling rate of the sensors used to fill the databases. However, the sheer amount of available data prevents the direct analysis of those time series. Furthermore, even the automated data-mining algorithms have a hard time scaling up to the task handling long time series. For this reason, one usually computes new representations of the time series. There can be two (non exclusive) goals for those representations:

- Dimensionality reduction. The representations should preserve as much as possible the underlying information. Usually, such representations allow to reconstruct time series as close as possible to the original time series (according to some distance measure). The difference between those representations

^ This work as been supported by grants from Région-Île-de-France. 
and the one used for compression is the need to enable some operations directly on those representation (for example computing distance measures).

- Information extraction. The representations should make the underlying information explicit. Those high-level representations must exhibit the relevant information as defined beforehand. This is akin to pattern matching and symbolic process-monitoring [10] where episodes of interest are detected and labeled according to a predefined set of shapes.

There has recently been a growing interest in symbolic representations of time series. The poster child of such representations being SAX (Symbolic Aggregate approXimation) 9] which enables many data-mining tasks. Symbolic representations fall clearly into the information extraction kind of time series representations, where episodes are associated to symbols interpretable according to the data-mining task. In this paper, we propose to learn those symbols without prior knowledge in order to build more relevant symbolic representations. The rest of this paper is organized as follows. Section 2 presents background and related work in time series symbolic representations and adaptive versus non adaptive representations. Section 3 presents our proposed Segmentation-Based Symbolic Representations (SBSR) with an emphasis on those based on piecewise constant segmentations (with linear model of order 0) SBSR-L0. In the same section, we propose a generic algorithm to compute SBSR and a specific algorithm for SBSR-L0. In sections 4, we evaluate the modeling accuracy of our algorithm. For space constraint reasons, we could only illustrate the results on our real-world dataset in this paper. However, more extensive evaluations, along with more detailed algorithms, are available in an expanded version of this paper 2. In section 5 we discuss the distance measures that can be defined over SBSR in order to take advantage of the accurate modeling for a better lower bounding distance measure. We present our conclusions and current research directions in section 6 .

\section{Background and Related Works}

As we have seen in section 1, time series databases are usually much too large to be tackled directly by data-mining algorithms. As far as this paper is concerned, the problem does not come from the number of time series in the database, but from the length of the time series. For this reason, we build representations of time series in order to reduce the dimensionality of the dataset, as it is a very common preprocessing step. A model for time series databases handling both the number of time series and the length of those time series is presented in 4. Many time series data-mining algorithms are based on a distance measure between time series, so defining such a distance measure is crucial when designing new time series representations, as we will see in section [5] Amongst the many time series representations already available, we will focus on those tackling issues the most related to our need for concise and expressive time series representations: 
1. SAX (Symbolic Aggregate approXimation), for the information extraction process of turning numerical time series into strings of symbols,

2. APCA (Adaptive Piecewise Constant Aggregation), for the advantages of locally adaptive representations with regard to conciseness / modeling accuracy tradedoff, when compared to its non-adaptive counterpart (Piecewise Aggregate Approximation).

The dataset of the univariate time series to be represented is defined as follows: Let $S=\left\{T S^{i}\right\}_{i \in\{1 \ldots M\}}$ be the dataset of $M$ time-series. Each of the time series is defined on the same time stamps: $T S^{i}=\left\{\left(v_{j}^{i}, d_{j}\right)\right\}_{i \in\{1 \ldots N\}}$ with $v_{j}^{i} \in \mathbb{R}$ and $d_{j} \in D, D$ being the temporal definition domain of the time series.

\subsection{SAX: Symbolic Aggregate Approximation}

The underlying principles of SAX are that the values taken by the normalized time series follow a normal distribution, and that the time series are "oversampled" according to the interesting patterns. Amongst the advantages of such a simple representation, is the fact that computational requierments for building such representations are minimal and that it is easy to compare representations of different time series if the episodes are of the same length. Another important asset of SAX is the distance measure that can be defined to lower bound the euclidean distance. SAX is based on the mean value of the time series over episodes of the same length. The sequence of those means is in fact another time series representation, but a numerical one, called Piecewise Aggregate Approximation (PAA).

\subsection{Adaptive Piecewise Constant Approximation Versus Piecewise Aggregate Approximation}

SAX is a kind of symbolic version of PAA, that is based on piecewise constant models (or linear models of order 0) over episodes of the same duration, built by quantizing the means according to a partition. PAA was introduced as an effective representation of time series in [7] where it was shown to enable dimensionality reduction and indexing of time series databases. As always, there is a tradeoff to be made between the accuracy of the representation and its conciseness. The accuray is often measured by the sum of square errors (SSE) and the conciseness by the complexity of the representation (i.e. the number of episodes for PAA).

Better accuracy/conciseness tradeoffs can be achieved thanks to $\operatorname{APCA}([5])$, an adaptive variant of PAA where episode length is locally adapted to the values taken by the time series. In search of time series symbolic representations that would yield the benefits of adaptive representations, that is better conciseness versus accuracy tradeoffs, we propose a new kind of symbolic representations based on segmentations. 


\section{SBSR: Segmentation-Based Symbolic Representations}

\subsection{Generic Framework for Symbolic Representations of Time Series}

With the notations presented in section 2, we defined the symbolic representations in [1] as follows:

- $E=\left\{e_{p}=\left(d_{p}, d_{p+1}\right)\right\}_{l \in\{1 \ldots P\}}$ is a set of $P$ episodes that is a partition of the time domain $D$.

$-\Lambda$ is a set of $K$ symbols.

- $S R(T S)=\left\{\left(e_{p}, \lambda_{p}, \ldots\right)\right\}_{p \in\{1 \ldots P\}}$ is a symbolic representation of $T S$ that allows to define a function : $E \rightarrow \Lambda, e_{p} \mapsto \lambda_{p}$. Each tuple of $S R(T S)$ is at least made of an episode and a symbol, but other information can be added, either numerical of symbolical in nature.

This very broad definition of symbolic representations encompasses a great number of symbolic representations. Different kinds of such symbolic representations are studied in [3], but we now present a new kind of Segmentation-Based Symbolic Representations (SBSR) that makes use of segmentation algorithms in order to compute the sets of episodes.

\subsection{Segmentation-Based Symbolic Representations}

Segmented models of time series can be defined as $\operatorname{Seg}(T S)=\left\{\left(e_{p}, \phi_{p}\right)\right\}$ where $\phi_{p}$ is a set of parameter values for the given model. PAA and APCA are both segmented models for linear models of order 0 , and linear models of order 1 are used in [6].

SBSR generalizes the quantization of the means in PAA that leads to SAX by defining clusters of parameters values. Let $\Lambda=\left\{\lambda_{k}\right\}_{k \in\{1 \ldots K\}}$ be the alphabet of symbols associated to $\Gamma=\left\{\gamma_{k}\right\}_{k \in\{1 \ldots K\}}$ the partition of the parameter space, $S B S R(T S)=\left\{\left(e_{p}, \lambda_{p}, \ldots\right)\right\}_{p \in\{1 \ldots P\}}$ is a symbolic representation such that $e_{p} \mapsto \lambda_{k} / \phi_{p} \in \gamma_{k}$ for a given segmentation of TS into $P$ segments $\operatorname{Seg}(T S)=\left\{\left(e_{p}, \phi_{p}\right)\right\}_{p \in\{1 \ldots P\}}$.

SBSR can be applied to any model but we now focus on the representation based on linear models of order 0 , that we call SBSR-L0.

\subsection{Segmentation-Based Symbolic Representations with Linear Models of Order 0}

SBSR-L0 are defined as follows:

$-\Lambda=\left\{\lambda_{k}\right\}_{k \in\{1 \ldots K\}}$ is the alphabet of symbols. Each $\lambda_{k}$ is related to a prototypical numerical value $\phi_{k}^{\prime} \in \mathbb{R}$ and an interval $I_{k}=\left\{\phi \in \mathbb{R} /\left(\phi-\phi_{k}^{\prime}\right)^{2}<\right.$ $\left.\left(\phi-\phi_{l}^{\prime}\right)^{2} \forall l \neq k\right\}$.

- $E=\left\{e_{p}=\left(d_{p}, d_{p+1}\right)\right\}_{p \in\{1 \ldots P\}}$ is the partition of $D$ into $P$ episodes.

- SBSR-L0 $(T S)=\left\{\left(e_{p}, \lambda_{p}\right)\right\}_{p \in\{1 \ldots P\}}$, where $\lambda_{p}$ is the symbol relating to the interval that contains $\overline{T S}\left[e_{p}\right]$, the mean value of $T S$ over the episode $e_{p}$. 
What we call restricted segmentation is a segmentation where the model parameters can only take values from a finite subset of the parameter space (here $\left.\left\{\phi_{k}^{\prime}\right\}_{k \in\{1 \ldots K\}}\right)$. Fig 1 shows the SBSR-L0 representation of the daily extract of the time series. It is important to note that the levels used for this extract are computed in order to represent the whole time series and not only this small extract, hence it is a daily extract of the SBSR-L0 representation of the whole time series and not SBSR-L0 representation of a daily extract.

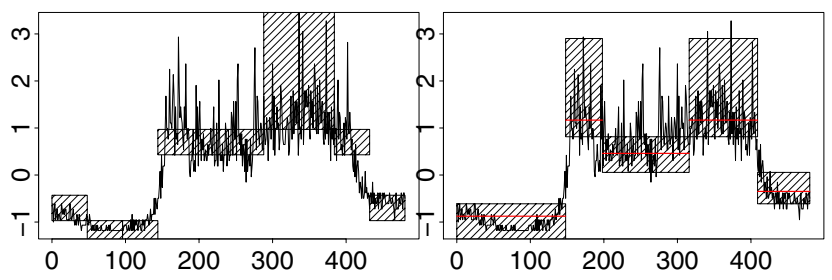

Fig. 1. Daily extract (over 480 points) of SAX and SBSR-L0 representations with 10 symbols

As we have seen in section 3.1 the most difficult part is often to devise efficient algorithms to compute $E$ and $\Lambda$ in order to maximize the modeling accuracy. However, it is possible to take advantage of the efficient segmentation algorithms to compute SBSR in general and especially SBSR-L0.

\subsection{Generic Algorithm for Computing SBSR}

The generic algorithm that we propose is a two-step iterative process much like the k-means [1] and E-M algorithms:

1. Compute initial segmentation $\operatorname{Seg}^{0}(T S)=\left\{\left(e_{p}{ }^{0}, \phi_{p}{ }^{0}\right)\right\}_{p \in\{1 \ldots P\}}$ of $T S$ into $P$ segments with the best algorithm affordable according to the number of points $N$.

2. Optimization of the interpretations of alphabet $\left\{\phi_{k}^{\prime s}\right\}$ for a given set of episodes computed in previous step $s$. We cluster the set of extracts of $\left\{T S\left[e_{p}^{s}\right]\right\}$ into $K$ clusters according to the clustering criterion and compute prototypical values $\left\{\phi_{k}^{s}\right\}$ that minimize the reconstruction error when each episode is associated to the prototypical value of its cluster.

3. Optimization of the set of episodes $E^{s+1}$ according to a given set of interpretations $\left\{\phi_{k}^{\prime s}\right\}$. We perform restricted segmentation of TS with model parameters values restricted to $\left\{\phi_{k}^{\prime s}\right\}$.

4. Repeat from step 2 until convergence

\section{Modeling Accuracy}

In order to provide simple distance measures between the time series of our datasets, we decided to compute only one set of episodes $E$ and one set of symbols 


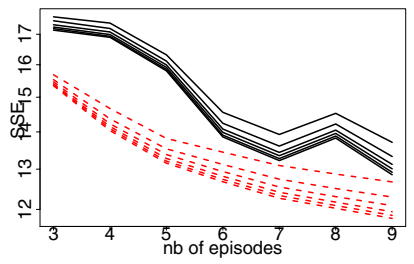

Fig. 2. SSE for numerical reconstructions from SAX and SBSR-L0 representations of our real-world dataset

and their interpretations for the whole dataset, and not specific sets for each of the time series. The modeling accuracy would have been even better with specific sets of episodes and alphabets of symbols. Given that SAX representations do not define a numerical reconstruction, we decided to assign to each SAX symbol a numerical value that is the mean value of the normal distribution over the associated interval.

The real-world dataset that we used to evaluate SBSR-L0 is based on urban traffic information sampled every 3 minutes by 423 sensors. The time series are normalized and the dataset is made of the 5000 daily extracts of 480 points from those time series. With notations in section 2, we have $M=5000$ and $N=480$. In Fig 2. we present the SSE for numerical reconstructions from SAX and SBSRL0 representations. Each curve represents the SSE for a number of symbols $(K \in\{4 \ldots 9\}$. It is important to notice that the SSE scale is logarithmic. The dahsed lines (resp. plain lines) are all thoses corresponding to SBSR-L0 (resp. SAX) representations. Within each of these groups the better results are of course those of the representations with the most symbols. It is interesting to note that SAX modeling is not always increasingly more accurate when more episodes are used for the representations.

\section{Distance Measures}

As we have seen in section 1, in order to be useful for data-mining tasks, representations of time series must enable the use of distance measures. Of course, the main advantage of symbolic representations is to enable specific manipulations using the domain semantic. A semantic distance between symbolic representations of time series would make use of an edit distance (or Levenshtein distance 8]) between the symbol strings and a semantic distance between each symbol.

However, it is often very important to be able to compare time series according to the underlying numerical data, for example by using the euclidean distance.

As we have just seen, the distance factor between symbols of SAX representations is based on the theoretical worst-case of mean values over the set of represented time-series extract. This pessimistic approach is good because it does not require any information from the represented time series and is not dependent on the datasets. However, it would be possible to lower bound more efficiently the euclidean distance with information about the mean values actually taken 
by the time series in the dataset. This also makes the case for representations both numerical and symbolical in nature. As for numerical computations such as distance measures, one cannot beat the accuracy of numerical information. From the smallest to the largest amount of numerical information that can be put into SBSR-L0, we have:

1. No additionnal information, as in SAX. Let us call the associated distance $D_{S A X}$.

2. Dataset global information. For each symbol, we store the actual minimal and maximal values of mean values taken by the time series extracts over episodes represented by the symbol. Let us call the associated distance $D_{g}$.

3. Time series local information. For each symbol and each of the time series, we store the actual minimal and maximal values of mean values taken by the time series extracts over episodes represented by the symbol. Let us call the associated distance $D_{l}$.

4. Episode local information, as in APCA. Let us call the associated distance $D_{A P C A}$.

It is obvious that we have $D_{S A X}\left(T S_{i 1}, T S_{i 2}\right) \leq D_{g}\left(T S_{i 1}, T S_{i 2}\right) \leq D_{l}\left(T S_{i 1}, T S_{i 2}\right) \leq$ $D_{A P C A}\left(T S_{i 1}, T S_{i 2}\right) \leq D\left(T S_{i 1}, T S_{i 2}\right), \forall\left(T S_{i 1}, T S_{i 2}\right) \in S \times S$.

The best tradeoff between the space requirements and efficient lower bounding is application dependent. In Fig 3 , we show the results of the evaluation of the distance functions over our real-world dataset (presented in section 4) for various values of $K$ and $P$.

We computed the mean thightness for all of the distances between each pair of time series in the dataset. We represented the tightness of 4 distance measures $\left(D_{S A X}, D_{g}, D_{l}, D_{A P C A}\right)$ on both SAX and SBSR-L0 representations. That should be 8 curves but we can see only 6 because $D_{S A X}$ and $D_{g}$ are extremely close to each other.

From the lowest (worst) to the highest (best), the curves that we can see are :

1. $D_{S A X}$ and $D_{g}$ for SAX

2. $D_{S A X}$ and $D_{g}$ for SBSR-L0

3. $D_{l}$ for SAX

4. $D_{l}$ for SBSR-L0

5. $D_{A P C A}$ for SAX

6. $D_{A P C A}$ for SBSR-L0

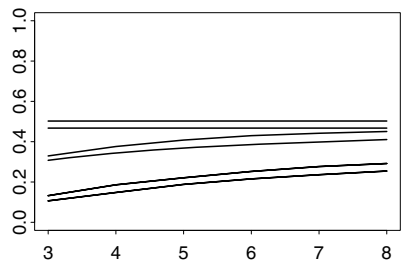

Fig. 3. Mean tightness of lower bounding by the various distance measures for 9 symboles and 3 to 10 episodes on our real-world dataset

\section{Conclusions and Future Works}

We have proposed SBSR, a new generic framework for locally symbolic and numeric/symbolic representations of time series based on segmentations and a generic algorithm to build those representations. 
Amongst SBSR, we focused on those based on piecewise constant models with prototypical values associated to symbols: SBSR-L0. We proposed an algorithm to build locally optimal SBSR-L0 and made experimental validations of the modeling accuracy of SBSR-L0 on a real-world dataset. We proposed various distance measures between SBSR-L0 representations, corresponding to different tradeoffs between space requirements and accuracy of the euclidean distance lower bounding and provided experimental results for those distances measures.

The SBSR framework allows to define numerous kinds of symbolic or numeric/symbolic representations of time series. When the information to extract concerns the local linear trends of time series, we ressort to SBSR based on clustering of trends in linear models of order 1 (SBSR-L1-T).

\section{References}

1. G. Hebrail and B. Hugueney. Symbolic representation of long time series. In Conference on Applied Statistical Models and Data Analysis (ASMDA), pages 537542, June 2001.

2. B. Hugueney. Expanded version of Adaptive Segmentation-Based Symbolic Representations of Time Series for BetterModeling and Lower Bounding DistanceMeasures. http://www.lamsade.dauphine.fr/hugueney/PKDD2006-Expanded.pdf.

3. B. Hugueney. Représentations symboliques de longues series temporelles. PhD thesis, LIP6, 2003.

4. B. Hugueney, G. Hébrail, and Y. Lechevallier. Computing summaries of time series databases with clustering and segmentation. Int. Fed. of Classification Societies, 2006.

5. E. Keogh, K. Chakrabarti, M. Pazzani, and S. Mehrotra. Locally adaptive dimensionality reduction for indexing large time series databases. SIGMOD Record (ACM Special Interest Group on Management of Data), 30(2):151-162, June 2001.

6. E. Keogh and M. J. Pazanni. An enhanced representation of time series which allows fast and accurate classification, clustering and relevance feedback. In D. Heckerman, H. Mannila, D. Pregibon, and R. Uthurusamy, editors, Proceedings of the Forth International Conference on Knowledge Discovery and Data Mining (KDD- 98). AAAI Press, 1998.

7. E. J. Keogh, K. Chakrabarti, M. J. Pazzani, and S. Mehrotra. Dimensionality reduction for fast similarity search in large time series databases. Knowledge and Information Systems Journal, 2000.

8. V. I. Levenshtein. Binary codes capable of correcting deletions, insertions and reversals. Soviet Physics Doklady., 10(8):707-710, Feb. 1966. Doklady Akademii Nauk SSSR, V163 No4 845-848 1965.

9. J. Lin, E. Keogh, S. Lonardi, and B. Chiu. A symbolic representation of time series, with implications for streaming algorithms. In Proceedings of the 8th ACM SIGMOD workshop on Research issues in data mining and knowledge discovery, pages 2-11. ACM Press, 2003.

10. P. L. Love and M. Simaan. Automatic recognition of primitive changes in manufacturing process signals. Pattern Recognition, 21(4):333-342, 1988.

11. J. MacQueen. Some methods for classification and analysis of multivariate observations. In Proceedings of the Fifth Berkeley Symposium on Mathematical Statistics and Probability, volume 1, pages 281-297, 1967. 